\title{
Turning on LIGHT
}

\author{
Steve W. Granger and Carl F. Ware \\ Division of Molecular Immunology, La Jolla Institute for Allergy and Immunology, \\ San Diego, California, USA
}

Commentary

See related article, pages $1771-1780$.

Address correspondence to: Carl F. Ware, Division of Molecular Immunology, La Jolla Institute for Allergy and Immunology,

10355 Science Center Drive, San Diego, California 92121, USA.

Phone: (858) 678-4660; Fax: (858) 558-3595; E-mail: carl_ware@liai.org.

J. Clin. Invest. 108:1741-1742 (2001). DOI:10.1172/JCI200114651.

The delicate balance between the proliferation and elimination of antigenactivated lymphocytes achieves homeostasis in peripheral lymphoid tissues. The TNF-related cytokines activate cellular differentiation, survival, and death pathways that orchestrate tissue development, organization, and homeostasis (1). Several members of the TNF superfamily of cytokines play opposing roles in lymphocyte homeostasis by enhancing effector cell activation and survival, or by cellular elimination through apoptosis.

Emerging evidence indicates that a recently defined member of the TNF superfamily, LIGHT (TNFSF14), plays a key role in $\mathrm{T}$ cell homeostasis. Studies by Wang et al. (2), in this issue of the JCI, and Shaikh et al. (3) reveal that sustained expression of LIGHT causes profound inflammation and loss of tolerance leading to autoimmune syndromes. These new findings validate LIGHT as an important $\mathrm{T}$ cell regulatory molecule and suggest its candidacy as a pharmaceutical target for diseases involving $\mathrm{T}$ cells.

LIGHT is structurally and functionally an integral member of the immediate TNF family, defined by a close structural homology and a communal pattern of receptor-ligand pairing with lymphotoxin- $\alpha \beta(\mathrm{LT} \alpha \beta), \mathrm{LT} \alpha$, and Fas ligand $(4,5)$. LIGHT is a type II transmembrane protein, produced by activated $T$ cells and immature dendritic cells, that signals through two distinct cellular receptors: the herpesvirus entry mediator (HVEM), which is expressed prominently on T cells, and the LT $\beta$ receptor (LT $\beta R$ ), which is expressed on stromal cells but absent from lymphocytes. LIGHT has been proposed to mediate $\mathrm{T}$ cell activation, survival, or death, and also - by analogy with $\mathrm{LT} \alpha \beta$ - to help organize lymphoid tis- sues (6). Indeed, results in tissue culture models appear to support a role for LIGHT in $\mathrm{T}$ cell activation $(7,8)$, although the responses of $\mathrm{T}$ cells to LIGHT signaling in vitro are rather subtle. The phenotype of mice expressing ectopic LIGHT is anything but subtle.

The studies by Wang et al. (2) and Shaikh et al. (3) demonstrate that constitutive expression of LIGHT leads to profound inflammation caused by activated T cells. Normally, LIGHT is transiently expressed on the surface of $\mathrm{T}$ cells following activation, but in the studies highlighted here, the proximal lck (2) or $C D 2$ (3) promoters drive the constitutive expression of LIGHT in $\mathrm{T}$ cells. At several months of age, both lines of LIGHT transgenic mice show lymphoid tissue abnormalities, including splenomegaly, lymphadenopathy, and pronounced inflammation in the intestine, consisting of expanded populations of conventional $\mathrm{CD}^{+}$and $\mathrm{CD}^{+}$ $\alpha \beta$ T cells. The inflamed intestines show signs of chronic processes including loss of goblet cells, distortion and hyperplasia of crypts, villous atrophy, and mononuclear cell infiltrates. Remarkably, an LT $\beta R-F c$ decoy receptor (a chimera of the receptor's ligand-binding domain for LIGHT fused with the Fc region of $\operatorname{IgG}$ that neutralizes both LIGHT and LT $\alpha \beta$ ) prevents colitis in a CD4 T cell-dependent transfer model (9). In this model, expression of $L T \alpha \beta$ in the recipient mice is not required for disease symptoms (10), implicating LIGHT as the relevant ligand. Thus, increased or sustained expression of LIGHT on activated $\mathrm{T}$ cells contributes to the induction and persistence of inflammation in the intestine.

However, the two studies observed significant differences in the phenotypes of the LIGHT transgenic mice $(2,3)$. Mice expressing LIGHT under the lck promoter exhibit extensive inflammation in the skin and hair follicles in addition to the intestine, whereas mice with $C D 2$ LIGHT fail to reproduce because of severely atrophied reproductive organs. The autoimmune nephritis, complete with anti-DNA antibodies, seen in lckLIGHT mice suggests that LIGHT induces a loss of tolerance to self-tissues. By contrast, the $\mathrm{B}$ cell compartment in the CD2-LIGHT transgenic mice is selectively expanded in the lamina propria of the small and large intestine,
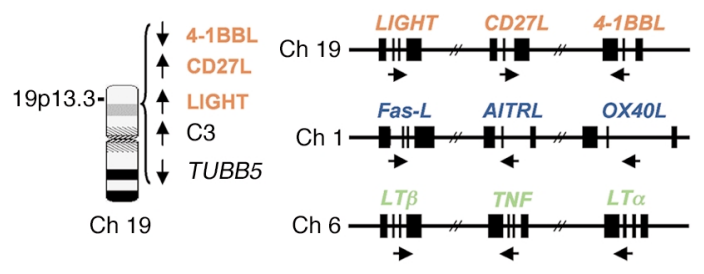

T cell activation/thymus Costimulation Costimulation Cell Death $T$ cell survival Memory $\mathrm{T}$ cells CD4 ${ }^{+}$progenitors, NK, NKT Cell Death CD4 ${ }^{+}$progenitors, NK, NKT

\section{Figure 1}

Organization of the TNF superfamily genes within the paralogous regions present on chromosomes 1, 6, and 19. Left panel: Diagram of the human chromosome 19p13.3 region containing the LIGHT genomic locus. Middle panel: Distribution of TNF paralogous superfamily gene clusters arranged from centromere to telomere. Arrows indicate gene transcriptional orientation, and solid blocks represent exons. LIGHT is $7.78 \mathrm{~kb}$ from C3 and about $79 \mathrm{~kb}$ from CD27L. CD27L is about $235 \mathrm{~kb}$ from 4-1BBL. Fas $L$ is separated from AITRL by $374 \mathrm{~kb}$, while AITRL and OX4OL are $134 \mathrm{~kb}$ apart. TNF is $2.9 \mathrm{~kb}$ from $L T \beta$ and $1.3 \mathrm{~kb}$ from $L T \alpha$. Right panel: activities of the TNF-related ligands on $T$ cells. 
with elevated serum IgA levels, despite B cell depletion in spleen and lymph nodes. These phenotypic differences are puzzling but most likely reflect differences in tissue-specific expression by these promoters, since $\mathrm{CD} 2$ is expressed in $\mathrm{T}$ and some B cells, whereas Lck is active in thymocytes and peripheral $T$ cells. Quantitative differences in ligand expression, and effects on different populations of cells may also help explain discrepancies between the two reports. In any case, both studies provide fresh insight into the function of LIGHT.

Wang et al. (2) provide additional evidence in their report that LIGHT is an effector molecule in the tissuedestructive phase of $\mathrm{T}$ cell-dependent autoimmune disease. They show that in NOD mice, which spontaneously develop insulin-dependent diabetes mellitus due to $T$ cell-dependent destruction of insulin-producing $\beta$ cells, the neutralization of LIGHT signaling via HVEM-Fc decoy receptor decreases the incidence of diabetes. This conclusion is supported in tumor and allograft models as well $(11,12)$.

Is the tissue destruction observed in the LIGHT transgenic mice due to nonspecific inflammation by activated $\mathrm{T}$ cells or true autoimmunity due to loss of tolerance? Interestingly, in both types of LIGHT transgenic mice, the thymi are small and contain few CD4/8 double-positive cells, the thymocytes that are normally subject to negative selection. In another report, blockade of LIGHT signaling in vitro and in vivo prevented negative selection induced by intrathymically expressed antigens, resulting in the rescue of thymocytes from apoptosis (13). Although speculation abounds, no other TNF family member but CD30 ligand has yet been confirmed as a factor in modulating negative selection. The present results $(2,3)$ suggest that LIGHT affects central differentiation processes critical for $T$ cell tolerance. Interestingly, the phenotype of LIGHT-deficient mice suggests a cooperative role for LIGHT and LT $\beta$ in lymphoid organogenesis and a costimulatory function for LIGHT in T cell activation (S. Scheu and K. Pfeffer, personal communication). An eager audience awaits the experiment designed to test whether central tolerance is affected by the absence of LIGHT. Animal models clearly demonstrate that dysregulated expression of the TNF-related cytokines leads to autoaggressive pathology. These new findings help clear the path to therapeutic interventions of autoimmune diseases.

More than half of the ligands in the TNF superfamily appear to be involved in regulating $\mathrm{T}$ cell homeostasis, which is reflected in the highly conserved genomic organization of these ligands (Figure 1). The LIGHT gene resides on human chromosome 19, adjacent to genes for CD27 ligand and 4-1BB ligand, which together forms a tripartite locus (14). The LIGHT locus is strikingly similar in organization to the TNF superfamily locus residing within the MHC on chromosome 6 and TNF superfamily members on chromosome 1 , which are recognized as paralogous regions (15). The genetic position and transcriptional orientation of LIGHT, $L T \beta$, and Fas ligand parallels their receptor-binding specificities. Where known, the TNF superfamily members in these paralogous gene clusters, such as the ligands for CD27, 4-1BB, and OX40 (see, for example, ref. 16), act as costimulatory molecules enhancing $\mathrm{T}$ lymphocyte activation and survival, or else they induce elimination of activated T cells, as has been well described for TNF and Fas ligand. The homeostatic signals are delivered by two distinct types of receptors: those with the death domain module, which activate caspases, and those that employ the TRAF adapters, which activate transcription of $\mathrm{Bcl}-\mathrm{xL}$ and other survival genes controlled by NF- $\kappa B$. In a variation of this theme, LT $\beta$ (as the LT $\alpha \beta$ complex) is necessary for differentiation of $\mathrm{CD} 4$ progenitors for lymph nodes and Peyer's patches, and NK and NK-T cells $(17,18)$. In contrast, BAFF and CD40 ligand, which map to distinct loci, are the primary mediators of $B$ cell survival and differentiation (19). No doubt the evolutionary conservation of the TNFrelated ligands dedicated to $\mathrm{T}$ cell homeostasis and linkage to antigen recognition molecules mirrors their importance in fine-tuning antigen recognition and immune tolerance.

\section{Acknowledgments}

The authors recognize the support provided in part by the US Public Health Service, NIH grants CA-69381, AI-03368, and AI-48073. This is publication 456 from the La Jolla Institute for Allergy and Immunology.
1. Locksley, R.M., Killeen, N., and, Lenardo, M.J. 2001. The TNF and TNF receptor superfamilies: integrating mammalian biology. Cell. 104:487-501.

2. Wang, J., et al. 2001. The regulation of $\mathrm{T}$ cell homeostasis and autoimmunity by $\mathrm{T}$ cell-derived LIGHT. J. Clin. Invest. 108:1771-1780.

3. Shaikh, R., et al. 2001. Constitutive expression of LIGHT on T cells leads to lymphocyte activation, inflammation and tissue destruction. J. Immunol. 167:6330-6337.

4. Mauri, D.N., et al. 1998. LIGHT, a new member of the TNF superfamily, and lymphotoxin $\alpha$ are ligands for herpesvirus entry mediator. Immunity. 8:21-30

5. Yu, K.Y., et al. 1999. A newly identified member of tumor necrosis factor receptor superfamily (TR6) suppresses LIGHT-mediated apoptosis. J. Biol. Chem. 274:13733-13736.

6. Fu, Y.-X., and Chaplin, D. 1999. Development and maturation of secondary lymphoid tissues. Annu. Rev. Immunol. 17:399-433.

7. Harrop, J.A., et al. 1998. Herpesvirus entry mediator ligand (HVEM-L), a novel ligand for HVEM/TR2, stimulates proliferation of T cells and inhibits HT29 cell growth. J. Biol. Chem. 273:27548-27556.

8. Tamada, K., et al. 2000. LIGHT, a TNF-like molecule, costimulates $\mathrm{T}$ cell proliferation and is required for dendritic cell-mediated allogeneic $\mathrm{T}$ cell response. J. Immunol. 164:4105-4110.

9. Mackay, F., et al. 1998. Both the lymphotoxin and tumor necrosis factor pathways are involved in experimental murine models of colitis. Gastroen terology. 115:1464-1475.

10. Corazza, N.S., Eichenberger, S., Eugester, H.P., and Muller, C. 1999. Non-lymphocyte derived tumor necrosis factor is required for induction of colitis in recombination activating gene (RAG) 2-/- mice upon transfer of CD4+ CD45RB high T cells. J. Exp. Med. 190:1479-1486.

11. Tamada, K, et al. 2000. Modulation of T-cell mediated immunity in tumor and graft-versus host disease models through the LIGHT co-stimulatory pathway. Nat. Med. 6:283-289.

12. Wu, Q., et al. 2001. Reversal of spontaneous autoimmune insulitis in nonobese diabetic mice by soluble lymphotoxin receptor. J. Exp. Med. 193:1327-1332.

13. Wang, J., et al. 2001. The critical role of LIGHT, a TNF family member, in T cell development. $J$. Immunol. 167:5099-5105

14. Granger, S.W., Butrovich, K.D., Houshmand, P., Edwards, W.R., and Ware, C.F. 2001. Genomic characterization of LIGHT reveals linkage to an immune response locus on chromosome 19p13.3 and distinct isoforms generated by alternate splic ing or proteolysis. J. Immunol. 167:5122-5128.

15. Flajnik, M.F., and Kasahara, M. 2001. Comparative genomics of the MHC: glimpses into the evolution of the adaptive immune system. Immunity. 15:351-362.

16. Rogers, P.R., Song, J., Gramaglia, I., Killeen, N., and Croft, M. 2001. OX40 promotes Bcl-xL and $\mathrm{Bcl}-2$ expression and is essential for longterm survival of CD4 $\mathrm{T}$ cells. Immunity. 15:445-455

17. Mebius, R.E., Rennert, P., and Weissman, I.L. 1997. Developing lymph nodes collect CD4+CD3- LTbeta+ cells that can differentiate to APC, NK cells, and follicular cells but not T or B cells. Immunity. 7:493-504.

18. Yoshida, H., et al. 2001. Expression of alpha(4)beta(7) integrin defines a distinct pathway of lymphoid progenitors committed to $\mathrm{T}$ cells, fetal intestinal lymphotoxin producer, NK, and dendritic cells. J. Immunol. 167:2511-2521.

19. Thompson, J.S., et al. 2001. BAFF-R, a newly identified TNF receptor that specifically interacts with BAFF. Science. 293:2108-2111. 